\title{
Therapeutic effect of budesonide, montelukast and azithromycin on post-infectious bronchiolitis obliterans in children
}

\author{
XIA CHEN, JUN-HUA SHU, YANG HUANG, ZHEN LONG and XIAO-QIN ZHOU \\ Department of Pediatric Pulmonology, Maternal and Child Health Hospital \\ of Hubei Province, Wuhan, Hubei 430070, P.R. China
}

Received September 30, 2019; Accepted May 29, 2020

DOI: $10.3892 /$ etm.2020.8983

\begin{abstract}
Optimal treatment options for post-infectious bronchiolitis obliterans (PIBO) have not yet been established. The present study retrospectively analyzed the effect of budesonide, montelukast and azithromycin on treating PIBO in children $<5$ years old.. Based on treatment regimen, the cohort was divided into group A and group B. Group A received a combination of budesonide, montelukast and azithromycin for at least 3 months and group B received unconventional treatment (budesonide for nebulization intermittently, prednisone, montelukast and antibiotics if necessary) compared with standard treatment. Tidal pulmonary function and symptoms assessment were performed at diagnosis and after 3 months of therapy. There were no significant differences in the sex, age, pulmonary function and symptoms assessment between groups A and B at diagnosis. However, following 3 months of treatment, the time to peak tidal expiratory flow as a proportion of expiratory time, and volume to peak expiratory flow as a proportion of exhaled volume in group A were significantly higher compared with those in group B. The respiratory rate in group A was significantly lower compared with group B. The symptoms assessment score in group A was significantly higher compared with that of group B. In conclusion, the present study demonstrates that combination therapy with budesonide, montelukast and azithromycin improves pulmonary function and respiratory symptoms in PIBO children $<5$ years old. The present study was retrospectively registered on March 22, 2020 with register no. YY202003-008-HB03.
\end{abstract}

\section{Introduction}

Bronchiolitis obliterans (BO) is an irreversible obstructive lung disease, which is characterized by subepithelial inflammation

Correspondence to: Dr Xia Chen, Department of Pediatric Pulmonology, Maternal and Child Health Hospital of Hubei Province, 745 Luo Yu Road, Wuhan, Hubei 430070, P.R. China

E-mail: chenxiawhu1@163.com

Key words: post-infectious bronchiolitis obliterans, budesonide, montelukast, azithromycin, children $<5$ years old and fibrotic narrowing of the bronchioles (1). A previous study published in 2011 reported that the overall prevalence of BO among all transplanted patients in the United States of America was 5.5\%, and $14 \%$ among patients with chronic graft-vs.-host disease (2). The presence of inflammation and fibrosis of the terminal and respiratory bronchioles leads to a narrowing or full obliteration of the airway lumen, as well as the chronic obstruction of air flow (3). The clinical manifestations of BO include persistent cough, wheezing and dyspnea, which seriously affect the health and quality of life of patients (4). The etiology of BO include infection, organ transplantation, connective tissue disease and exposure to toxic fumes, and the most common cause of BO in children is infection $(5,6)$. Post-infectious bronchiolitis obliterans (PIBO) often occurs secondary to severe lower respiratory tract infections (7). PIBO is a rare disease and as there are no national or international databases on PIBO, its incidence is unpredictable but is more frequent among certain populations such as Argentinians, Native Americans and native Koreans (8). The development of PIBO is associated with adenovirus, measles virus, Mycoplasma pneumonia, influenza virus and respiratory syncytial virus $(9,10)$. The diagnostic rate of PIBO in children has improved in recent years, with the application of high-resolution computerized tomography (CT), however suitable and efficacious treatment options, particularly for long-term chronic management, have not yet been established (11).

The etiology of BO in children is not completely understood and PIBO is especially common in children $(9,12)$. At present, there is no precise treatment for PIBO (7). It is argued that long-term use of glucocorticoid for treating PIBO cannot reverse the pre-existing airway obstruction, but can reduce airway hyperresponsiveness and bronchial stenosis (13). A previous study suggested that glucocorticoids could slow the progression of BO (14). Some studies demonstrated that pulse corticotherapy may be a safe and alternative approach to prolonged systemic oral corticotherapy in children with BO $(13,15)$. Considering the side effects of long-term systemic glucocorticoid application, whether inhaled corticosteroids (ICS) can be used as an alternative treatment for PIBO remains to be elucidated. Some studies demonstrated that ICS demonstrated treatment efficacy in patients with BO, following hematopoietic stem cell transplantation $(16,17)$. ICS is the most effective drug for the control of airway inflammation (18). 
The selected drugs of ICS include budesonide, fluticasone and betamethasone dipropionate. Budesonide is a moderately fat-soluble glucocorticoid and its fat solubility is lower compared with that of fluticasone and beclomethasone dipropionate (19). As there are special anatomical structures, such as serous layer and mucilage in the airway, fat-soluble drugs do not dissolve easily in the mucus and can be easily removed by the mucociliary structures (20). The relatively low-fat-soluble budesonide is more likely to enter the airway via the serous layer of the mucosa surface in the airway (21).

Steroid therapy is the cornerstone of BO treatment. However, the side effects of long-term systemic administration of glucocorticoids and ICS merit investigation of an alternative treatment for PIBO $(22,23)$. A previous study demonstrated that the combination therapy of fluticasone, azithromycin and montelukast decreased total corticosteroid exposure in eight patients with $\mathrm{BO}$, following hematopoietic stem cell transplantation $(24,25)$. Other studies have also demonstrated that azithromycin and montelukast had treatment efficacy in BO $(26,27)$. However, the treatment efficacy of ICS combined with azithromycin and montelukast in children with PIBO remains poorly understood.

The present study retrospectively analyzed the effect of budesonide, montelukast and azithromycin on the treatment of PIBO in children $<5$ years old, aiming to provide some guidance for their clinical application in the treatment of PIBO.

\section{Materials and methods}

Subjects. A total of 53 patients (mean age, 21 months; age range, 6 months- 4 years) treated in the Pediatric Pulmonology department of the Maternal and Child Health Hospital of Hubei Province (Wuhan, China) between May 2015 and May 2019, were retrospectively analyzed in the present study. The disease duration prior to diagnosis of PIBO was 2-20 months. All the cases included in the study were in line with the diagnostic criteria of PIBO (7) as follows: i) Recurrent or persistent cough, wheezing, shortness of breath for $>6$ weeks following acute infection, and no response to bronchodilator; ii) respiratory symptoms that were severely disproportionate to the chest $\mathrm{X}$-ray findings; iii) unilateral hyperlucent lung observed via a chest radiography examination; iv) bronchial wall thickening, bronchiectasis, pulmonary atelectasis or mosaic perfusion observed on a pulmonary $\mathrm{CT}$; v) obstructive ventilatory disorder demonstrated by lung function tests; and vi) exclusion of other obstructive diseases, such as asthma, congenital ciliary dyskinesia, immune deficiency and pancreatic fibrocystic changes. The exclusion criteria were as follows: i) Complications from other pulmonary diseases, such as asthma, pneumonia or pulmonary tuberculosis; and ii) patients with severe respiratory difficulties and transcutaneous oxygen saturation $<88 \%$. The present study was approved by the Ethics Committee of the Maternal and Child Health Hospital of Hubei Province (Wuhan, China). Written informed consent was obtained from all of the participants' parents/guardians.

Treatment and grouping of patients. Based on different treatment regimens and parental compliance, enrolled patients were divided into group A $(n=33)$ and group $B(n=20)$. Group A received a combination of budesonide, montelukast and azithromycin (1 mg budesonide solution inhaled twice daily, $4 \mathrm{mg}$ montelukast orally once daily and $5 \mathrm{mg} / \mathrm{kg}$ azithromycin orally once daily for the first 3 days of every week) for at least 3 months. Group B received an unconventional treatment (budesonide for nebulization intermittently, prednisone, montelukast and antibiotics if necessary), in which patients were treated intermittently in accordance with symptoms. Patients in group B received intermittent budesonide or prednisone due to parents' rejection for long-term glucocorticoid treatment. There was no bias or specific criteria used in the grouping of patients. The age, duration and symptoms at the time of enrollment were similar for the two groups. The patients were followed up by telephone every 2 weeks and came to the hospital once a month for consultation. If the symptoms became worse or severe, they will make a consultation by telephone. Two children in group B developed coughing and severe dyspnea due to cold and were not included in the final analysis. All children with $\mathrm{BO}$ presented with coughing during the entire study period.

Pulmonary function test. Pulmonary function tests were performed at diagnosis and after 3 months of treatment. Children underwent the tidal pulmonary function test by Master Screen Paed (Care Fusion). The nasopharyngeal secretions of patients were cleared before testing and the patients were treated with oral $10 \%$ chloral hydrate $(50-70 \mathrm{mg} / \mathrm{kg}$, maximum not exceeding $2 \mathrm{~g}$ ) for sedation (28) and no adverse reactions were found. The main test parameters of tidal pulmonary function, reflecting obstructive ventilatory disorder included: time to peak tidal expiratory flow as a proportion of expiratory time (TPTEF/TE), volume to peak expiratory flow as a proportion of exhaled volume (VPEF/VE), ratio of inspiratory time and expiratory time (TI/TE), respiratory rate (RR) and the tidal volume per kilogram of body weight $(\mathrm{VT} / \mathrm{Kg})$.

Symptoms assessment test. At present, there is no specific questionnaire to assess the symptoms of PIBO in children $<5$ years old (12). The test for respiratory and asthma control in kids (TRACK) is a validated quantitative tool for assessing asthma control in preschool-aged children (29). TRACK can be completed fully by the primary carer of the child (30). TRACK includes details on the frequency of respiratory symptoms (wheezing, cough and shortness of breath), night-time awakenings and activity limitation in the past 4 weeks; the rescue medication used in the past 3 months and; the oral corticosteroid used in the previous year. Using TRACK and considering the characteristics of PIBO in children $<5$ years old, the questionnaire used in the present study only included the first three questions of TRACK (Table I). The score of each questionnaire ranged from 0-20. The better the symptoms were controlled, the higher the score was. The questionnaire was performed at diagnosis and after 3 months of treatment.

Statistical analyses. Normally distributed continuous variables are presented as the means \pm standard deviation (SD), while non-normally distributed continuous data are presented as medians and interquartile ranges (25-75th). The paired Student's t-test or the Wilcoxon signed-rank test was performed to compare the difference between the diagnosis and following 3-month treatment results within groups. The 
Table I. Details of questionnaire for symptom assessment of patients with post-infectious bronchiolitis obliterans.

\begin{tabular}{|c|c|c|c|c|c|}
\hline \multirow[b]{2}{*}{ Questions } & \multicolumn{5}{|c|}{ Score } \\
\hline & 20 & 15 & 10 & 5 & 0 \\
\hline $\begin{array}{l}\text { 1. During the past } 4 \text { weeks, how often } \\
\text { did the child suffer from respiratory } \\
\text { problems, such as cough, wheezing or } \\
\text { shortness of breath? }\end{array}$ & Not at all & Once or twice & Once every week & $\begin{array}{l}2 \text { or } 3 \text { times } \\
\text { a week }\end{array}$ & $\begin{array}{l}4 \text { or more times } \\
\text { a week }\end{array}$ \\
\hline $\begin{array}{l}\text { 2. During the past } 4 \text { weeks, how often } \\
\text { was the child's sleep disrupted by } \\
\text { respiratory problems? }\end{array}$ & Not at all & Once or twice & Once every week & $\begin{array}{l}2 \text { or } 3 \text { times } \\
\text { a week }\end{array}$ & $\begin{array}{l}4 \text { or more times } \\
\text { a week }\end{array}$ \\
\hline $\begin{array}{l}\text { 3. During the past } 4 \text { weeks, how limited } \\
\text { was the child in performing normal } \\
\text { activities by respiratory problems? }\end{array}$ & Not at all & Slightly & Moderately & Quite a lot & Extremely \\
\hline
\end{tabular}

The questionnaire was performed based on the test for respiratory and asthma control in kids (29).

independent sample t-test or the Mann-Whitney U test was conducted for comparison between groups A and B. $\chi^{2}$ test was performed for the comparison of sex distribution between the two groups. Statistical analyses were performed using the SPSS software v.19.0 (IBM Corp.). $\mathrm{P}<0.05$ was considered to indicate a statistically significant difference.

\section{Results}

Clinical characteristics of patients. A total of 46 patients suffered from serious pulmonary infections during the first two years of life (28 patients in group A and 18 in group B). The main clinical manifestations were recurrent coughing, wheezing and shortness of breath following severe respiratory infection. Moist rales and wheezing were heard in the lung of most children. Of the total patient cohort, 18 patients were infected with adenovirus, 11 with measles virus, 9 with Mycoplasma pneumoniae and 4 with respiratory syncytial virus. No specific pathogen was found in 11 patients. Pulmonary CT demonstrated mosaic perfusion and patchy ground-glass opacity in almost all patients. Bronchial wall thickening and bronchiectasis were observed in 25 patients; localized and transparent lung was found in 21 patients; pulmonary atelectasis was found in 17 patients; emphysema was found in 12 patients; and bronchial mucus suppository was found in 10 patients. There were no significant differences in sex, age, pulmonary function and symptom assessment between group A and group B at the time of PIBO diagnosis (Table II).

For patients in group A, a significant increase in height and weight was observed after 3 months treatment compared with the time of diagnosis $(\mathrm{P}<0.001$; Table III). However, no changes in liver and kidney function was found following treatment, demonstrated by no significant changes observed in the levels of alanine aminotransferase, aspartate aminotransferase, serum creatinine and blood urea nitrogen $(\mathrm{P}>0.05$; Table III).

Improved pulmonary function at diagnosis and after 3-month therapy in group A. Following the 3-month treatment period,
TPTEF/TE and VPEF/VE were significantly higher in group A compared with group $\mathrm{B}$ (both $\mathrm{P}=0.002$; Fig. $1 \mathrm{~A}$ ). $\mathrm{RR}$ was significantly lower in group A $(24.72 \pm 4.04)$ compared with group $\mathrm{B}(27.30 \pm 4.13)(\mathrm{P}=0.03$; Fig. 1A). However, there were no significant differences in TI/TE and VT/Kg between group A and group $\mathrm{B}(\mathrm{P}>0.05$; Fig. $1 \mathrm{~A})$. In the present study, the value of TPTEF/TE were 13.46 \pm 3.91 in group A and 12.48 \pm 3.24 in group B, respectively, and the value of $\mathrm{VPEF} / \mathrm{VE}$ were $18.35 \pm 3.53$ in group $\mathrm{A}$ and $17.80 \pm 3.17$ in group $\mathrm{B}$, respectively. After 3-month treatment, TPTEF/TE and VPEF/VE were significantly increased compared with their values at PIBO diagnosis in group $\mathrm{A}$ (both $\mathrm{P}<0.001$; Fig. 1B), but were slightly decreased in group $B$ although, this did not reach statistical significance $(\mathrm{P}=0.096$ and $\mathrm{P}=0.097$; Fig. $1 \mathrm{C})$. There were no significant differences for $\mathrm{VT} / \mathrm{Kg}$ or $\mathrm{TI} / \mathrm{TE}$ before and after treatment (Fig. 1). RR was decreased significantly compared with its level at PIBO diagnosis in group $\mathrm{A}(\mathrm{P}<0.001$; Fig. 1B) but was slightly increased in group $\mathrm{B}(\mathrm{P}=0.083$; Fig. $1 \mathrm{C})$.

Comparison of symptoms at diagnosis and after 3-month therapy in group $A$ and group B. All patients' parents completed the questionnaire at the time of PIBO diagnosis and following the 3-month treatment period (Table I). The symptom assessment test score was significantly increased after 3-month treatment compared with the assessment at PIBO diagnosis in group A $(\mathrm{P}<0.001$; Table IV) and the test score was significantly decreased after 3-month treatment in group B $(\mathrm{P}<0.001$; Table IV). Specifically, the score for all the three TRACK questions included in the present study were significantly increased in group $\mathrm{A}(\mathrm{P}<0.001, \mathrm{P}=0.001$ and $\mathrm{P}=0.008$, respectively; Table IV) and significantly decreased in group $\mathrm{B}(\mathrm{P}=0.011, \mathrm{P}=0.022$ and $\mathrm{P}=0.001$, respectively; Table IV). The test score in group A was significantly higher compared with that in group B after 3-month treatment $(\mathrm{P}<0.001$; Fig. 2). A more accurate scale for evaluating the symptoms of children with PIBO needs to be designed. Pulmonary CT may change very slowly in BO, so it is generally not used as a short-term evaluation index (7). Pulmonary 
Table II. Clinical and demographic characteristics of the subjects.

\begin{tabular}{lccc}
\hline Characteristics & Group A & Group B & P-value \\
\hline Number of patients (n) & $\mathrm{n}=33$ & $\mathrm{n}=20$ \\
Sex (n) & & 14 & 0.645 \\
Male & 25 & 6 & \\
Female & 8 & $22.05 \pm 11.17$ & \\
Age (months) & $20.78 \pm 10.27$ & & 0.677 \\
Pulmonary function & & $9.06 \pm 1.45$ & 0.547 \\
VT/Kg & $8.81 \pm 1.48$ & $12.48 \pm 3.24$ & 0.577 \\
TPTEF/TE & $13.46 \pm 3.91$ & $17.80 \pm 3.17$ & 0.799 \\
VPEF/VE & $18.35 \pm 3.53$ & $26.66 \pm 4.50$ & 0.619 \\
RR & $27.47 \pm 4.85$ & $0.59 \pm 0.12$ & 0.120 \\
TI/TE & $0.55 \pm 0.11$ & & 0.965 \\
Symptoms assessment & & $5(5-10)$ & 0.899 \\
Respiratory symptoms & $5(5-10)$ & $10(5-15)$ & 0.852 \\
Night-time awakenings & $10(5-15)$ & $15(10-15)$ & 0.888 \\
Activity limitation & $15(10-15)$ & $30(25-35)$ & \\
Total score & $30(20-40)$ & &
\end{tabular}

Data are presented as means \pm standard deviation or medians and interquartile ranges. VT/Kg, tidal volume per kilogram of body weight; TPTEF/TE, time to peak tidal expiratory flow as a proportion of expiratory time; VPEF/VE, volume to peak expiratory flow as a proportion of exhaled volume; RR, respiratory rate; TI/TE ratio of inspiratory time and expiratory time.

Table III. Comparison of height, weight, liver and kidney function before and after treatment in group A of patients with PIBO.

\begin{tabular}{lrrr}
\hline Features & $\begin{array}{c}\text { PIBO } \\
\text { diagnosis }\end{array}$ & $\begin{array}{l}\text { 3 months } \\
\text { treatment }\end{array}$ & P-value \\
\hline Height (cm) & $80.18 \pm 7.82$ & $82.57 \pm 6.46$ & $<0.001$ \\
Weight (kg) & $11.25 \pm 2.36$ & $12.06 \pm 2.04$ & $<0.001$ \\
ALT (U/l) & $16.20 \pm 2.08$ & $16.34 \pm 2.09$ & 0.429 \\
AST (U/l) & $20.34 \pm 2.52$ & $20.19 \pm 2.58$ & 0.417 \\
SCR (umol/l) & $40.31 \pm 3.74$ & $40.14 \pm 3.52$ & 0.266 \\
BUN (mmol/l) & $3.81 \pm 0.37$ & $3.79 \pm 0.38$ & 0.232 \\
\hline
\end{tabular}

Data were are presented as means \pm standard deviation. ALT, alanine aminotransferase; AST, aspartate aminotransferase; SCR, serum creatinine; BUN, blood urea nitrogen; PIBO, post-infectious bronchiolitis obliterans.

CT examination 6 months or one year after therapy and high-resolution CT scanning indicated marked improvements in group A compared with group B (Fig. 3).

Concomitant treatments and adverse events. For complicated infection, cough and sputum, antibiotic treatment was performed in the present study. In addition, a small number of children taking azithromycin will have gastrointestinal symptoms, nausea, vomiting and diarrhea, and these symptoms can be relieved without special treatment (31). Rescue medications were administered for children with breathing difficulties in the present study. Oxygen inhalation, bronchodilator inhalation, application of systemic glucocorticoids was performed, including ventilator-assisted breathing if necessary. However, according the inclusion criteria (blood oxygen saturation is not less than $88 \%$ ), patients were excluded in the present study, if the patient had difficulty breathing and had a significant decrease of blood oxygen. The treatment regimens used in the present study were based on adult treatment and the clinicians' experience. The drugs administered were safe and reliable and no special adverse effects were observed during the study.

\section{Discussion}

Oral administration of small doses of azithromycin can exert anti-inflammatory and immunomodulatory effects and reduce pulmonary infection and lung injury $(32,33)$. Azithromycin can inhibit the activity of neutrophils and reduce the secretion of cytokines [interleukin (IL)-6, IL-8 and tumor necrosis factor], which can improve the pulmonary function of patients with BO (34). Leukotriene receptor antagonists can inhibit airway inflammation (35). Montelukast has been demonstrated to show a high treatment efficacy on patients with BO after transplantation (27). Consistent with these studies, the findings of the present study suggested that the combined application of budesonide, azithromycin and montelukast for at least 3 months improved the pulmonary function and respiratory symptoms in children with PIBO. In addition, no abnormal changes in liver and kidney function after treatment were found.

The measurement of pulmonary function plays a significant role in judging the severity of disease, evaluating clinical curative effect and predicting prognosis (36). The most commonly used pulmonary function test in children 


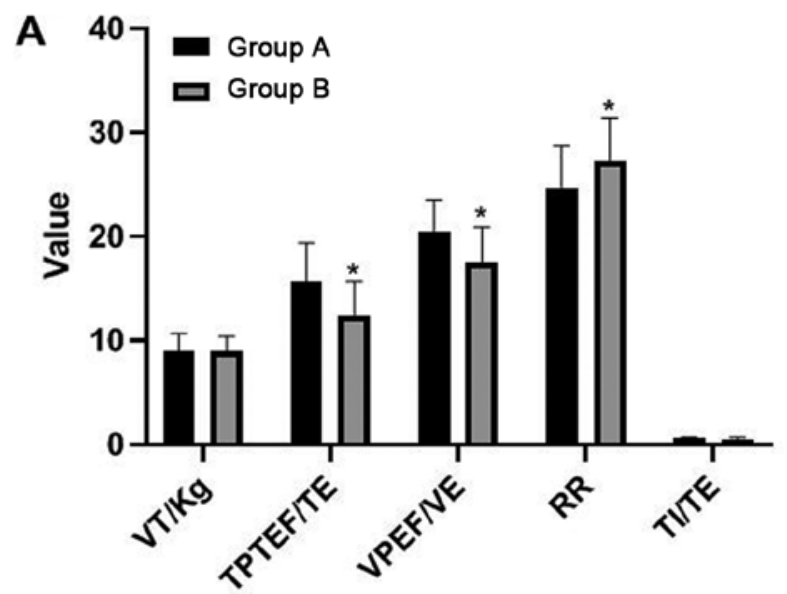

\section{Parameters}

\section{B}

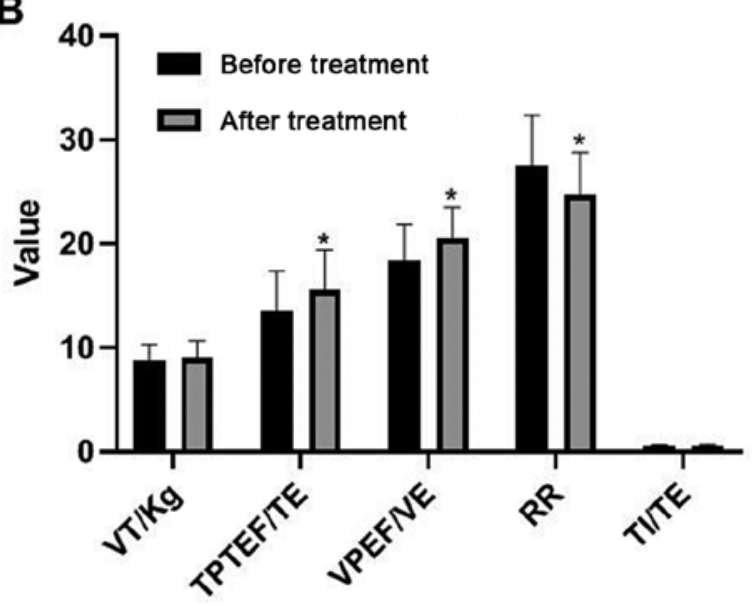

Parameters

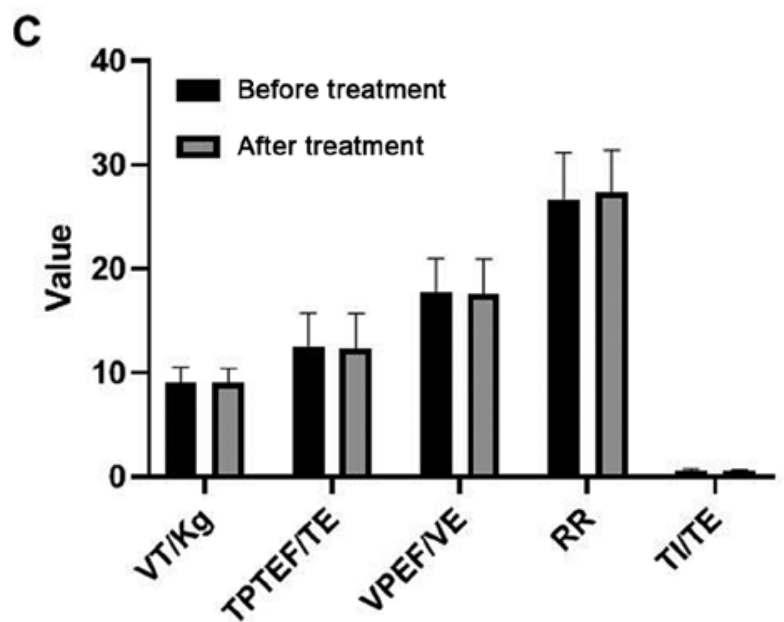

Parameters

Figure 1. Comparison of pulmonary function test in patient groups with post-infectious bronchiolitis obliterans. (A) Comparison of pulmonary function between group A and group B after 3-month treatment. ${ }^{*} \mathrm{P}<0.05$ vs. Group A. (B) Comparison of pulmonary function in group A before and after treatment. ${ }^{*} \mathrm{P}<0.05$ vs. before treatment. (C) Comparison of pulmonary function in group B before and after treatment. VT/Kg, tidal volume per kilogram of body weight; TPTEF/TE time to peak tidal expiratory flow as a proportion of expiratory time; VPEF/VE, volume to peak expiratory flow as a proportion of exhaled volume; $\mathrm{RR}$, respiratory rate; TI/TE, ratio of inspiratory time and expiratory time.

$<5$ years old is the tidal breath pulmonary function test $(28,37,38)$. TPTEF/TE and VPEF/VE are important parameters reflecting obstructive ventilatory disorder with a normal range of $28-55 \%$. According to different degree of obstruction, they are divided into mild obstruction (28-23\%), moderate obstruction (22-15\%) and severe obstruction $(<15 \%)$ (39). In the present study, the presence of moderate-to-severe airway obstruction at PIBO diagnosis was observed. In the present study, both TPTEF/TE and VPEF/VE were significantly increased after 3-month treatment in group $\mathrm{A}$, suggesting that the combined application of budesonide, azithromycin and montelukast was effective. Children are metabolically active; however, their lung capacity is small. The breathing frequency increases when obstructive or restrictive ventilation is impaired (40). The present study demonstrated that RR was significantly reduced after treatment in group A, but not in group B. TI/TE decreases when there is obstruction in the lower respiratory tract, due to increased expiratory resistance. The decline in $\mathrm{VT} / \mathrm{Kg}$ usually indicates restrictive lung disease, but it also exists in moderate-to-severe obstructive pulmonary disease (41). The findings of the present study demonstrated no statistically significant difference in TI/TE and $\mathrm{VT} / \mathrm{Kg}$ in the two groups, however were both slightly increased in group A and slightly decreased in group B. Williams et al (42) reported that treatment with fluticasone, azithromycin and montelukast and steroid pulse therapy may halt pulmonary decline in new-onset $\mathrm{BO}$ in the majority of patients and permit reductions in systemic steroid exposure. Consistent with this, the results of the present study indicated that the pulmonary function of children with PIBO was improved after treatment with budesonide, montelukast and azithromycin. In the current study, the majority of children suffered from serious pulmonary infections during the first two years of life, which was consistent with a previous study (18). Early intervention may help improve lung function (7). At present, there are few studies on tidal breath pulmonary function in infants and young children $(23,24)$. Thus, more research on the tidal breath pulmonary function test is imperative. 
Table IV. Change of PIBO symptoms assessment test following therapy.

A, Group A

\begin{tabular}{lccr}
\hline Questions & PIBO diagnosis & 3 months treatment & P-value \\
\hline Q1. Respiratory symptoms & $5(5-10)$ & $10(5-15)$ & $<0.001$ \\
Q2. Night-time awakenings & $10(5-15)$ & $10(10-15)$ & 0.001 \\
Q3. Activity limitation & $15(10-15)$ & $15(10-15)$ & 0.008 \\
Total of the three questions & $30(20-40)$ & $35(30-43$ & $<0.001$ \\
\hline
\end{tabular}

B, Group B

\begin{tabular}{lccr}
\hline Questions & PIBO diagnosis & 3 months treatment & P-value \\
\hline Q1. Respiratory symptoms & $5(5-10)$ & $5(5-5)$ & 0.011 \\
Q2. Night-time awakenings & $10(5-15)$ & $5(5-10)$ & 0.022 \\
Q3. Activity limitation & $15(10-15)$ & $10(5-10)$ & 0.001 \\
Total of the three questions & $30(25-35)$ & $20(20-25)$ & $<0.001$ \\
\hline
\end{tabular}

Data are presented medians and interquartile ranges. PIBO, post-infectious bronchiolitis obliterans.

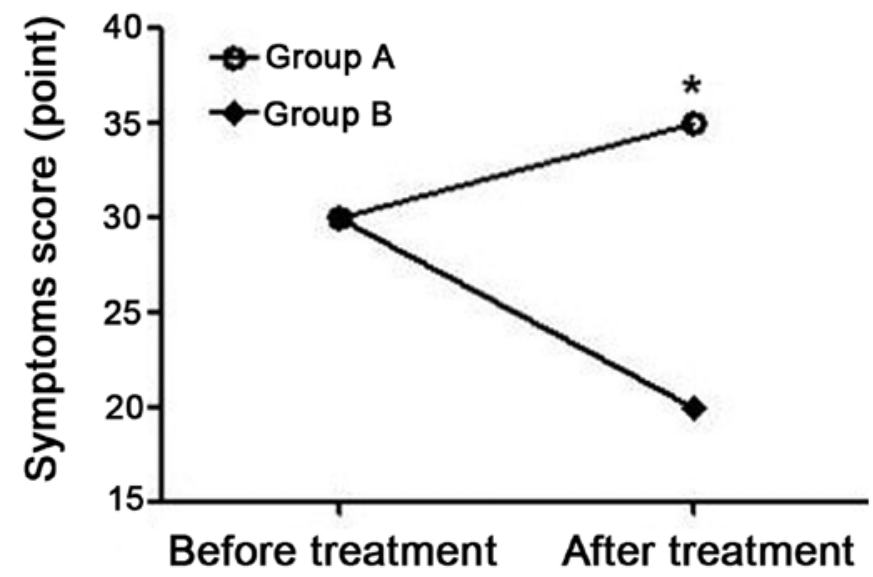

Figure 2. Comparison of symptoms assessment test after 3-month therapy between group A and group B patients with post-infectious bronchiolitis obliterans. The symptoms were assessed by the test for respiratory and asthma control in kids. $\mathrm{P}<0.05$ vs. Group B before treatment.

In addition to pulmonary function, symptoms assessment is another important indicator for evaluating clinical treatment effect (43). Using TRACK and considering the characteristics of PIBO in children, the questionnaire was applied. In the present study, the symptoms assessment test score was increased significantly after 3-month treatment in group A, but not in group B. Zhang et al (44) reported that clinical symptoms were significantly improved in patients with PIBO undergoing long-term nebulization treatment. Consistent with this, the present study reported that the symptoms were significantly controlled after treatment with budesonide, montelukast and azithromycin in patients with PIBO, but became worse without regular treatment.

The present study has some limitations. The sample size is relatively small and future clinical studies with larger cohorts are required to confirm the findings of the present study.

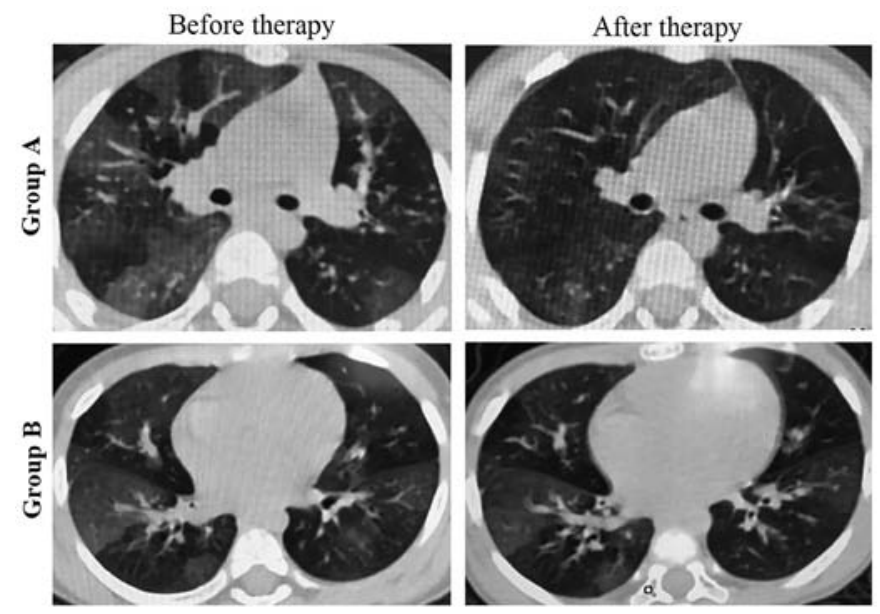

Figure 3. Pulmonary computerized tomography examination before and 1 year after therapy in patients from group A and group B with post-infectious bronchiolitis obliterans.

In conclusion, the present study demonstrated that combination therapy with budesonide, montelukast and azithromycin improved pulmonary function and respiratory symptoms in children $<5$ years old with PIBO. Further studies are required to assess the long-term outcomes and survival benefits of the combination treatment used in the present study.

\section{Acknowledgements}

Not applicable.

\section{Funding}

This study was funded by a grant from the Hubei Province Health and Family Planning Scientific Research Project (grant no. WJ2018H0150). 


\section{Availability of data and materials}

All data generated or analyzed during this study are included in this published article.

\section{Authors' contributions}

$\mathrm{XC}$, JHS, YH, ZL and XQZ performed the experiments and analyzed the data. XC designed the study and wrote the manuscript. All authors have read and approved the manuscript.

\section{Ethics approval and consent to participate}

The present study was approved by the Ethics Committee of the Maternal and Child Health Hospital of Hubei Province (Wuhan, China) (approval no. YY202003-008-HB03). Written informed consent was provided by the guardians/parents of all the participants.

\section{Patient consent for publication}

Not applicable.

\section{Competing interests}

The authors declare that they have no competing interests.

\section{References}

1. Barker AF, Bergeron A, Rom WN and Hertz MI: Obliterative bronchiolitis. N Engl J Med 370: 1820-1828, 2014.

2. Au BK, Au MA and Chien JW: Bronchiolitis obliterans syndrome epidemiology after allogeneic hematopoietic cell transplantation. Biol Blood Marrow Transplant 17: 1072-1078, 2011.

3. Hogg JC, Pare PD and Hackett TL: The contribution of small airway obstruction to the pathogenesis of chronic obstructive pulmonary disease. Physiol Rev 97: 529-552, 2017.

4. Sarria EE, Mundstock E, Machado DG, Mocelin HT, Fischer GB, Furlan SP, Antonello ICF, Stein R, Mattiello R, et al: Health-related quality of life in patients with bronchiolitis obliterans. J Pediatr (Rio J) 94: 374-379, 2018.

5. Moonnumakal SP and Fan LL: Bronchiolitis obliterans in children. Curr Opin Pediatr 20: 272-278, 2008.

6. Murtagh P, Giubergia V, Viale D, Bauer G and Pena HG: Lower respiratory infections by adenovirus in children. Clinical features and risk factors for bronchiolitis obliterans and mortality. Pediatr Pulmonol 44: 450-456, 2009.

7. Jerkic SP, Brinkmann F, Calder A, Casey A, Dishop M, Griese M, Kurland G, Niemitz M, Nyilas S, Schramm D, et al: Postinfectious bronchiolitis obliterans in children: Diagnostic workup and therapeutic options: A workshop report. Can Respir J 2020: 5852827, 2020.

8. Smith KJ and Fan LL: Insights into post-infectious bronchiolitis obliterans in children. Thorax 61: 462-463, 2006.

9. Colom AJ, Teper AM, Vollmer WM and Diette GB: Risk factors for the development of bronchiolitis obliterans in children with bronchiolitis. Thorax 61: 503-506, 2006.

10. Rosewich M, Zissler UM, Kheiri T, Voss S, Eickmeier O, Schulze J, Herrmann E, Dücker RP, Schubert R and Zielen S: Airway inflammation in children and adolescents with bronchiolitis obliterans. Cytokine 73: 156-162, 2015.

11. Colom AJ and Teper AM: Post-infectious bronchiolitis obliterans. Pediatr Pulmonol 54: 212-219, 2019.

12. Champs ND, Lasmar LMLBF, Camargos PAM, Marguet C, Fischer GB and Mocelin HT: Post-infectious bronchiolitis obliterans in children. J Pediat-Brazil 87: 187-198, 2011 (In English, Portuguese).
13. Tomikawa SO, Adde FV, da Silva Filho LV, Leone C and Rodrigues JC: Follow-up on pediatric patients with bronchiolitis obliterans treated with corticosteroid pulse therapy. Orphanet J Rare Dis 9: 128, 2014.

14. Moran TJ and Hellstrom HR: Bronchiolitis obliterans: An experimental study of the pathogenesis and the use of cortisone in modification of the lesions. AMA Arch Pathol 66: 691-707, 1958.

15. Ratjen F, Rjabko O and Kremens B: High-dose corticosteroid therapy for bronchiolitis obliterans after bone marrow transplantation in children. Bone Marrow Transplant 36: 135-138, 2005.

16. Bergeron A, Chevret S, Chagnon K, Godet C, Bergot E, Peffault de Latour R, Dominique S, de Revel T, Juvin K, Maillard N, et al: Budesonide/Formoterol for bronchiolitis obliterans after hematopoietic stem cell transplantation. Am J Respir Crit Care Med 191: 1242-1249, 2015

17. Kim SW, Rhee CK, Kim YJ, Lee S, Kim HJ and Lee JW: Therapeutic effect of budesonide/formoterol, montelukast and $\mathrm{N}$-acetylcysteine for bronchiolitis obliterans syndrome after hematopoietic stem cell transplantation. Respir Res 17: 63, 2016.

18. Li YN, Liu L, Qiao HM, Cheng H and Cheng HJ: Post-infectious bronchiolitis obliterans in children: A review of 42 cases. BMC Pediatr 14: 238, 2014.

19. Sobande PO and Kercsmar CM: Inhaled corticosteroids in asthma management. Respir Care 53: 625-634, 2008.

20. Elliot ER, Theodoraki A, Jain LR, Marshall NJ, Boffito M, Baldeweg SE and Waters LJ: Iatrogenic Cushing's syndrome due to drug interaction between glucocorticoids and the ritonavir or cobicistat containing HIV therapies. Clin Med (Lond) 16: 412-418, 2016.

21. Dalby C, Polanowski T, Larsson T, Borgstrom L, Edsbacker S and Harrison TW: The bioavailability and airway clearance of the steroid component of budesonide/formoterol and salmeterol/fluticasone after inhaled administration in patients with COPD and healthy subjects: A randomized controlled trial. Respir Res 10: 104, 2009.

22. Williams KM: How I treat bronchiolitis obliterans syndrome after hematopoietic stem cell transplantation. Blood 129: 448-455, 2017.

23. Verleden GM, Vos R, Dupont L, Van Raemdonck DE, Vanaudenaerde BM and Verleden SE: Are we near to an effective drug treatment for bronchiolitis obliterans? Expert Opin Pharmaco 15: 2117-2120, 2014.

24. Williams KM, Cheng GS, Pusic I, Jagasia M, Burns L, Ho VT, Pidala J, Palmer J, Johnston L, Mayer S, et al: Fluticasone, azithromycin, and montelukast treatment for new-onset bronchiolitis obliterans syndrome after hematopoietic cell transplantation. Biol Blood Marrow Transplant 22: 710-716, 2016.

25. Norman BC, Jacobsohn DA, Williams KM, Au BK, Au MA, Lee SJ, Moravec CK and Chien JW: Fluticasone, azithromycin and montelukast therapy in reducing corticosteroid exposure in bronchiolitis obliterans syndrome after allogeneic hematopoietic SCT: A case series of eight patients. Bone Marrow Transplant 46: 1369-1373, 2011

26. Gottlieb J, Szangolies J, Koehnlein T, Golpon H, Simon A and Welte T: Long-term azithromycin for bronchiolitis obliterans syndrome after lung transplantation. Transplantation 85: 36-41, 2008.

27. Verleden GM, Verleden SE, Vos R, De Vleeschauwer SI, Dupont LJ, Van Raemdonck DE and Vanaudenaerde BM: Montelukast for bronchiolitis obliterans syndrome after lung transplantation: A pilot study. Transpl Int 24: 651-656, 2011.

28. Nguyen TT, Hoo AF, Lum S, Wade A, Thia LP and Stocks J: New reference equations to improve interpretation of infant lung function. Pediatr Pulmonol 48: 370-380, 2013.

29. Murphy KR, Zeiger RS, Kosinski M, Chipps B, Mellon M, Schatz M, Lampl K, Hanlon JT and Ramachandran S: Test for respiratory and asthma control in kids (TRACK): A caregiver-completed questionnaire for preschool-aged children. J Allergy Clin Immunol 123: 833-839.e9, 2009.

30. Liu AH, Zeiger R, Sorkness C, Mahr T, Ostrom N, Burgess S, Rosenzweig JC and Manjunath R: Development and cross-sectional validation of the childhood asthma control test. J Allergy Clin Immunol 119: 817-825, 2007.

31. Diemert DJ: Prevention and self-treatment of traveler's diarrhea. Clin Microbiol Rev 19: 583-594, 2006.

32. Ruttens D, Verleden SE, Vandermeulen E, Bellon $H$, Vanaudenaerde BM, Somers J, Schoonis A, Schaevers V, Van Raemdonck DE, Neyrinck A, et al: Prophylactic azithromycin therapy after lung transplantation: Post hoc analysis of a randomized controlled trial. Am J Transplant 16: 254-261, 2016. 
33. Wang X, Liu C, Wang M, Zhang YI, Li H and Liu G: Clinical features of post-infectious bronchiolitis obliterans in children undergoing long-term azithromycin treatment. Exp Ther Med 9: 2379-2383, 2015.

34. Verleden GM, Vanaudenaerde BM, Dupont LJ and Van Raemdonck DE: Azithromycin reduces airway neutrophilia and interleukin-8 in patients with bronchiolitis obliterans syndrome. Am J Respir Crit Care Med 174: 566-570, 2006.

35. Okunishi K and Peters-Golden M: Leukotrienes and airway inflammation. Biochim Biophys Acta 1810: 1096-1102, 2011.

36. Bevacqua BK: Pre-operative pulmonary evaluation in the patient with suspected respiratory disease. Indian J Anaesth 59: 542-549, 2015.

37. Fuchs O, Latzin $P$, Thamrin C, Stern G, Frischknecht P, Singer F, Kieninger E, Proietti E, Riedel T and Frey U: Normative data for lung function and exhaled nitric oxide in unsedated healthy infants. Eur Respir J 37: 1208-1216, 2011.

38. Schmalisch G, Wilitzki S and Wauer RR: Differences in tidal breathing between infants with chronic lung diseases and healthy controls. BMC Pediatr 5: 36, 2005

39. Chen J, Liu X, Du W, Srivastava R, Fu J, Zheng M, Zhou J and McGrath E: Pulmonary function testing in pediatric pneumonia patients with wheezing younger than 3 years of age. Global pediatric health 6: 2333794X19840357, 2019.
40. O'Donnell DE, Elbehairy AF, Berton DC, Domnik NJ and Neder JA: Advances in the evaluation of respiratory pathophysiology during exercise in chronic lung diseases. Front Physiol 8: 82, 2017.

41. Fiorino S, Bacchi-Reggiani L, Detotto E, Battilana M, Borghi E, Denitto C, Dickmans C, Facchini B, Moretti R, Parini S, et al: Efficacy of non-invasive mechanical ventilation in the general ward in patients with chronic obstructive pulmonary disease admitted for hypercapnic acute respiratory failure and $\mathrm{pH}<7.35$ : A feasibility pilot study. Intern Med J 45: 527-537, 2015.

42. Williams KM, Cheng GS, Pusic I, Jagasia M, Burns L, Ho VT, Pidala J, Palmer J, Johnston L, Mayer S, et al: Fluticasone, Azithromycin, and montelukast treatment for new-onset bronchiolitis obliterans syndrome after hematopoietic cell transplantation. Biol Blood Marrow Transplant 22: 710-716, 2016.

43. Ranu H, Wilde M and Madden B: Pulmonary function tests. Ulster Med J 80: 84-90, 2011.

44. Zhang XM, Lu AZ, Yang HW, Qian LL, Wang LB and Zhang XB: Clinical features of postinfectious bronchiolitis obliterans in children undergoing long-term nebulization treatment. World J Pediatr 14: 498-503, 2018.

This work is licensed under a Creative Commons Attribution-NonCommercial-NoDerivatives 4.0 International (CC BY-NC-ND 4.0) License. 